\title{
Cognition of Schizophrenic Inpatients and Healthy Individuals: Casual-Comparative Study
}

\author{
S. Mohammad Moosavi ${ }^{1}$, Parivash Shirvani ${ }^{2}$, Mani B. Monajemi ${ }^{3, *}$ \\ ${ }^{1}$ Mazandaran University of Medical Sciences, Sari, Iran \\ ${ }^{2}$ Department of Clinical Psychology, Azad University of Sari, Sari, Iran \\ ${ }^{3}$ Department of Psychology, University of Tehran, Tehran, Iran
}

\section{Email address:}

mani.b.monajemi@warwickgrad.net (M. B. Monajemi), mani.b.monajemi@ut.ac.ir (M. B. Monajemi)

${ }^{*}$ Corresponding author

\section{To cite this article:}

S. Mohammad Moosavi, Parivash Shirvani, Mani B. Monajemi. Cognition of Schizophrenic Inpatients and Healthy Individuals: CasualComparative Study. American Journal of Psychiatry and Neuroscience. Vol. 5, No. 1, 2017, pp. 10-15. doi: 10.11648/j.ajpn.20170501.13

Received: August 29, 2016; Accepted: February 9, 2017; Published: March 2, 2017

\begin{abstract}
Introduction: Aim of current study is to compare cognitive condition of schizophrenic inpatients of psychiatric hospital and healthy individuals. Materials and Methods: This study is a practical research and with respect to nature and method is casual-comparative research. Statistical Society of this society consisted of 73 inpatients schizophrenic patients, who were admitted to Zare psychiatric hospital between 2015-2016. Sample Size Determination was done via Krejcie and Morgan Table and sample size reduced to 60 patients. In order to choose sample, random sampling method was used. Field data collection and library were used regarding obtaining data; GHQ28 and Wechsler Adult Intelligence Scale were used. Reliability of the test was 0.77 according to Cronbach's alpha. We assessed hypotheses of this study via SPSS software and univariate t-analysis. Results and Conclusion: A result of the study is demonstrative of meaningful difference between similarities, picture completion and information between case group and control group. However, no meaningful difference was noticed in aforementioned subtests with respect to duration of this disorder.
\end{abstract}

Keywords: Cognition, Cognitive Psychology, GHQ28, Schizophrenia, Wechsler Adult Intelligence Scale

\section{Introduction}

Among psychiatric disorders, psychosis and more specifically schizophrenia maintain significant importance among mental health professionals. Schizophrenia contains wide spectrum of cognitive, social and emotional deficiencies and lead to major malfunctioning in afflicted individuals [1].

Emil Kraepelin was one of the pioneers who categorized schizophrenia as specific disorder; due to early onset of this disorder Kraepelin called it youth psychosis. The term schizophrenia was firstly coined by Bleuler [2].

Schizophrenia is a chronic and severe mental disorder that affects how a person thinks, feels, and behaves. People with schizophrenia may seem like they have lost touch with reality. Although schizophrenia is not as common as other mental disorders, the symptoms can be very disabling.

Signs and Symptoms

Symptoms of schizophrenia usually start between ages 16 and 30. In rare cases, children have schizophrenia too.

The symptoms of schizophrenia fall into three categories: positive, negative, and cognitive.

Positive symptoms: "Positive" symptoms are psychotic behaviors not generally seen in healthy people. People with positive symptoms may "lose touch" with some aspects of reality. Symptoms include:

Hallucinations

Delusions

Thought disorders (unusual or dysfunctional ways of thinking)

Movement disorders (agitated body movements)

Negative symptoms: "Negative" symptoms are associated with disruptions to normal emotions and behaviors. Symptoms include:

"Flat affect" (reduced expression of emotions via facial 
expression or voice tone)

Reduced feelings of pleasure in everyday life

Difficulty beginning and sustaining activities

Reduced speaking

Cognitive symptoms: For some patients, the cognitive symptoms of schizophrenia are subtle, but for others, they are more severe and patients may notice changes in their memory or other aspects of thinking. Symptoms include:

Poor "executive functioning" (the ability to understand information and use it to make decisions), Trouble focusing or paying attention, Problems with "working memory" (the ability to use information immediately after learning it)

In recent years reviews have yield a reconsideration of some widely cited, but poorly supported by evidence, aspects of schizophrenia epidemiology. The first is the notion that schizophrenia has universal incidence across cultures and countries. The second is the belief that schizophrenia distributes itself equality in males and females. Taken together these beliefs could be conceptualized as schizophrenia is an equalitarian disorder, and schizophrenia is an exceptional disorder. It is puzzling that these two interrelated beliefs are usually cited as evidence for a biological origin of the disease, when most diseases in medicine do vary across cultures, countries, and gender [3].

Cognitive Dysfunction among Schizophrenic Patients:

Cognitive dysfunction is a core feature of schizophrenia. Deficits are moderate to severe across several domains, including attention, working memory, verbal learning and memory, and executive functions. These deficits pre-date the onset of frank psychosis and are stable throughout the course of the illness in most patients. Over the past decade, the focus on these deficits has increased dramatically with the recognition that they are consistently the best predictor of functional outcomes across outcome domains and patient samples. Recent treatment studies, both pharmacological and behavioral, suggest that cognitive deficits are malleable. Other research calls into question the meaningfulness of cognitive change in schizophrenia [4]. According to recent meta-analysis on cognitive deficits of patients with schizophrenia evidenced by the comparison with normal control cases, has confirmed the stability of the results found in the previous work. These findings show a generalized presence of cognitive impairment among the patients with schizophrenia. These results cannot be considered free of the potential bias that only controlled studies with positive results are available in the published evidence, while all those with negative results are not traceable. The real possibility of such a bias, should make us consider that the results obtained in this meta-analysis might be, in some degree, inflated by an underrepresentation of negative results [5].

It is clear that it is not possible to assess all detrimental aspects of schizophrenia in one study. Thus, in current study we mainly focused on cognitive problems of this group of patients. Screening and identifying cognitive problems related to this malady may assist clinicians in order to approach this disorder with more insight about its detrimental consequences.

\section{Materials and Methods}

This study is a practical research and with respect to nature and method is casual-comparative research. Statistical Society of this society consisted of 73 inpatients schizophrenic patients, who were admitted to Zare psychiatric hospital between 2015-2016. Sample Size Determination was done via Krejcie and Morgan Table and sample size reduced to 60 patients. In order to choose sample, random sampling method was used. Field data collection and library were used regarding obtaining data; GHQ28 and Wechsler Adult Intelligence Scale were used. Reliability of the test was 0.77 according to Cronbach's alpha. We assessed hypotheses of this study via SPSS software and univariate t-analysis

- Inclusion Criteria:

- Schizophrenic patients in Zare psychiatric hospital.

- Age range of patients' family members was between 20-50.

- Participants signed consent forms in order to participate in study.

- Exclusion Criteria:

- History of drug abuse, reported and confirmed by patient or urine test.

- Having other psychiatric disorders, which was diagnosed by psychiatrist.

- Reluctance to participate in study.

- Tools

Wechsler Adult Intelligence Scale-Revised

- Overview

The Wechsler intelligence scales were developed by Dr. David Wechsler, a clinical psychologist with Bellevue Hospital. His initial test, the Wechsler-Bellevue Intelligence Scale, was published in 1939 and was designed to measure intellectual performance by adults. Wechsler constructed the WBIS based on his observation that, at the time, existing intelligence tests for adults were merely adaptations of tests for children and had little face validity for older age groups.

Since 1939, three scales have been developed and subsequently revised, to measure intellectual functioning of children and adults. The Wechsler Adult Intelligence ScaleIII (WAIS-III) is intended for use with adults. The Wechsler Intelligence Scale for Children-III (WISC-III) is designed for children ages 6 - 16, while the Wechsler Preschool and Primary Scale of Intelligence-R (WPPSI-R) is designed for children age 4 - $61 / 2$ years.

- The WAIS-III

The WAIS-III is the 1997 revision of the test originally published in 1955 and first revised in 1981. The WAIS-III contains the following scales:

- Verbal Scales

- Information: 28 items on a variety of information adults have presumably had opportunities to acquire in our culture. No specialized or academic information included; however, some of the items cover quite sophisticated information.

- Comprehension: 18 items that require examinee to 
explain what should be done in certain circumstances, the meaning of proverbs, why certain societal practices are followed, and so forth. The test measures practical judgement, common sense, and the ability to understand and adapt to social customs. Score on each item varies (0-2 pts) according to the degree to which the response describes the most pertinent aspects of the question.

- Arithmetic: 20 arithmetic problems similar to those encountered in elementary math courses. Problems are administered orally and must be solved without paper and pencil. In addition to math knowledge, test measures concentration and systematic problem-solving ability.

- Similarities: 19 items requiring examinee to describe how two given things are alike. Score on each item varies according to the degree to which the response describes a general property primarily pertinent to both items in the pair. Measures concrete, functional, and abstract concept formation.

- Digit Span: Two parts, Digits forward and digits backwards. Examinee required to repeat 3 - 9 digits forward and 2 - 9 digits backwards. Measures shortterm memory, attention, and concentration.

- Vocabulary: 66 words of increasing difficulty are presented orally and visually. Examinee required to define the words. Score (0-2) based on sophistication of definition. Measures verbal knowledge and concept formation.

- Letter-Number Sequencing (Optional Test): Examiner presents combinations of letters and numbers, from 2 to nine letter-number combinations. Examinee must repeat each series by, first, repeating the numbers in ascending order, then the letters in alphabetical order (e.g., 9-L-2A; correct response is 2-9-A-L). Measures "working memory," the ability to simultaneously recall and organize stimuli of different, similar types. This also is a standard test on the Wechsler Memory Scale-III.

- Performance Scales

- Coding-Digit Symbol: Numbers 1 - 7 are paired with symbols on a key presented to examinee. Examinee has 120 seconds to go through a grid of 90 numbers and place the correct symbol above each number. Measures visual-motor speed and complexity, motor coordination. There are two additional, optional extensions of the coding test that measure the examinees skills in learning the coding process after completing the initial task.

- Picture Completion: 25 cards, each containing a picture having a part missing. Examinee must identify the missing part. Measures ability to observe details and recognize specific features of the environment (I.e., whole to part discrimination). Also measures performance in deliberately focusing attention.

- Block Design: Perhaps the butt of more jokes than any other WAIS scale! Included in the test are nine red and white square blocks and a spiral booklet of cards showing different color designs that can be made with the blocks. The examinee must arrange the blocks to match the design formed by examiner or shown on cards. In addition to being scored for accuracy, each item is scored for speed as well. Measures spatial problem-solving and manipulative abilities, and part to whole organization.

- Picture Arrangement: Eleven items. Each item consists of 3 to 6 cards containing pictures. The examinee must arrange the pictures from left to right to tell the intended story. Again, both accuracy and speed are scored. Partial credit is given for alternate, but less commonly given arrangements to some items.

- Measures nonverbal reasoning and sequencing skills, and grasp of social cause and effect (also known as social intelligence).

- Matrix Reasoning: A new test on the WAIS-III. Examinee is presented with a series of design with a part missing. Examinee chooses the missing part that will complete the design, from five choices. Measures nonverbal analytical reasoning.

- Object Assembly (Optional Test): Four items, each item being a "cut up" object, like a puzzle. Examinee must correctly assemble the parts of the puzzle. Measures visual-motor problem-solving and organizational abilities, and visual anticipation skills.

- Symbol Search (Optional test): Examinee must match one or two symbols shown on the left column with the same symbol/s in the right column of each page in the supplemental test booklet. Measures organization accuracy and processing speed

- Purpose: Designed as a comprehensive test of cognitive ability for adults.

- Population: Ages 16 and over.

- Score: Verbal IQ, Performance IQ, and Full Scale IQ.

- Time: (60-90) minutes.

- Author: David Wechsler.

- Publisher: The Psychological Corporation.

- Description: The Wechsler Adult Intelligence ScaleRevised (WAIS-R) is a general test of intelligence, which Wechsler defined as, "... the global capacity of the individual to act purposefully, to think rationally, and to deal effectively with his environment." In keeping with this definition of intelligence as an aggregate of mental aptitudes or abilities, the WAIS-R consists of 11 subtests divided into two parts, verbal and performance.

- Scoring: The WAIS-R consists of six verbal subtests and five performance subtests. The verbal tests are: Information, Comprehension, Arithmetic, Digit Span, Similarities, and Vocabulary. The Performance subtests are: Picture Arrangement, Picture Completion, Block Design, Object Assembly, and Digit Symbol. The scores derived from this test are a Verbal IQ (VIQ), a Performance IQ (PIQ), and a Full Scale IQ (FSIQ). The FSIQ is a standard score with a mean of 100 and a standard deviation of approximately 15 .

- Reliability: Corrected split-half reliability coefficients for Verbal IQ (.95 to.97) and Full Scale IQ (.96 to.98), 
and their respective standard errors of about 2 points, are quite acceptable. The reliability of Performance IQ is excellent, averaging.93, although the value of.88 at ages 16 to 17 is not ideal. Subtest reliability coefficients average values exceed. 80 for 9 of the 11 subtests. Only Picture Arrangement (.74) and Object Assembly (.68) fall short of expectations. Test-retest reliability coefficients affirm the excellent reliability of the Verbal and Full Scales, and show Performance IQ to be quite acceptable (.89 to.90). Test-retest coefficients for the subtests confirm the reliability of all tasks except Object Assembly and Picture Arrangement.

- Validity: The manual for this revised edition does not provide new validity data, but refers interested persons to the manual for the original WAIS.

- Norms: The total sample, comprising 1,880 individuals, was carefully stratified on the variables of sex, race (white-nonwhite), geographic region, occupational group, educational attainment, and urban-rural residence. The nine age groups ranged in size from 160 to 300 .

- Suggested Uses: The WAIS-R is recommended for use in clinical, educational, and research settings [6,7].

- GHQ (General Health Questionnaire): Developed in the 1970s, the General Health Questionnaire is a method to quantify the risk of developing psychiatric disorders. This instrument targets two areas - the inability to carry out normal functions and the appearance of distress - to assess well-being in a person.

- The format of the full GHQ is 60-item test with a four-point scale for each response. The test exits in several alternate forms: GHQ-30 (30 items), GHQ-28 (28-items), GHQ-12 (12 items).

- The reported Cronbach alpha coefficient for the GHQ is a range of 0.82 to 0.86 . The instrument is considered as reliable and has been translated into 38 different languages. When correlated with the global quality of life scale, the GHQ showed negative correlation. This demonstrates the inverse relationship with an increase in distress leading to a decrease in quality of life. (https://www.statisticssolutions.com/general-healthquestionnaire-ghq/)

- According to Malakouti and Colleagues (2007), GHQ28 was an internally consistent measure. Cronbach's alpha, split-half coefficients and test-retest reliability were $0.9,0.89$ and 0.58 respectively. Four factors were extracted using factor analysis: "depression," "psychosocial activity," "anxiety," and "somatic." Using receiver operating curve (ROC) analysis, the optimum cutoff score for the GHQ-28 in this group was $19 / 20$ (sensitivity 0.83 , specificity 0.76 ). Using a loading of 0.6 or greater, a short form of the instrument $(\mathrm{GHQ}-15)($ alpha $=0.9)$ was derived and correlated well with the longer form of the scale $(r=0.97)$. Using ROC analysis, the optimum cutoff score was $10 / 11$ (sensitivity 0.83 , specificity 0.69) [8].

\section{Results}

Data regarding gender distribution can be noticed in Table 1.

Table 1. Gender Distribution Data.

\begin{tabular}{llll}
\hline Group & Gender & Frequency & Percentage \\
\hline \multirow{2}{*}{ Control } & Male & 18 & 30 \\
& Female & 12 & 20 \\
\multirow{2}{*}{ Case } & Male & 19 & 32 \\
& Female & 11 & 18 \\
Total & & 60 & $\% 100$ \\
\hline
\end{tabular}

Data regarding educational status can be noticed in Table 2 .

Table 2. Educational Status Data.

\begin{tabular}{llll}
\hline Group & Educational Status & Frequency & Percentage \\
\hline \multirow{4}{*}{ Control } & Lower than High-School Degree & 12 & 20 \\
& High-School Degree and Upper & 11 & 18 \\
& Bachelor Degree and Upper & 7 & 12 \\
& Lower than High-School Degree & 17 & 28 \\
Case & High-School Degree and Upper & 8 & 14 \\
& Bachelor Degree and Upper & 5 & 8 \\
Total & & 60 & $\% 100$ \\
\hline
\end{tabular}

Data regarding marital status can be noticed in Table 3 .

Table 3. Data regarding marital status.

\begin{tabular}{llll}
\hline Group & Gender & Frequency & Percentage \\
\hline Control & Single & 15 & 25 \\
& Married & 15 & 25 \\
Case & Single & 16 & 27 \\
& Married & 14 & 23 \\
Total & & 60 & $\% 100$ \\
\hline
\end{tabular}

Data regarding duration of disease can be noticed in Table-4.

Table 4. Data regarding Disease duration.

\begin{tabular}{lll}
\hline Duration & Frequency & Percentage \\
\hline Up to 2 years old & 6 & 20 \\
2-5 year old & 11 & 37 \\
Older than 5 year old & 13 & 43 \\
Total & 30 & $\% 100$ \\
\hline
\end{tabular}

Regarding assessment of normal distribution of data we used Kolmogorov-Smirnov test. Data can be noticed in Table 5.

Table 5. Assessment of Normal distribution of Data.

\begin{tabular}{lllll}
\hline Group & Variables & $\mathbf{z}$ & Error & p-value \\
\hline \multirow{6}{*}{ Control } & Similarities & $0 / 965$ & $0 / 05$ & $0 / 309$ \\
& Information & $0 / 762$ & $0 / 05$ & $0 / 607$ \\
& Block Design & $0 / 861$ & $0 / 05$ & $0 / 449$ \\
& Picture Arrangement & $0 / 576$ & $0 / 05$ & $0 / 114$ \\
& IQ & $0 / 647$ & $0 / 05$ & $0 / 797$ \\
& Similarities & $1 / 03$ & $0 / 05$ & $0 / 232$ \\
& Information & $0 / 655$ & $0 / 05$ & $0 / 785$ \\
& Block Design & $0 / 376$ & $0 / 05$ & $0 / 999$ \\
& Picture Arrangement & $0 / 978$ & $0 / 05$ & $0 / 294$ \\
& IQ & $0 / 636$ & $0 / 05$ & $0 / 191$ \\
\hline
\end{tabular}

According to Table 6, p-value is lower than 0.05 and there is meaningful difference between score of similarities subtest between case group and control group; score of similarities subtest is higher in control group. According to Table 6, p- 
value is lower than 0.05 and there is meaningful difference between score of Information subtest between case group and control group; score of Information subtest is higher in control group. According to Table 6, p-value is lower than 0.05 and there is meaningful difference between score of Block Design subtest between case group and control group; score of Block Design subtest is higher in control group. According to Table 6, p-value is lower than 0.05 and there is meaningful difference between score of Picture Arrangement subtest between case group and control group; score of Picture Arrangement subtest is higher in control group. According to Table 6, p-value is lower than 0.05 and there is meaningful difference between score of $I Q$ subtest between case group and control group; score of IQ subtest is higher in control group.

Table 6. Results of t-Test with respect to Wechsler subtests.

\begin{tabular}{|c|c|c|c|c|c|c|c|}
\hline Group & Variables & Number of Participants & Mean & SD & t & $\alpha$ & p-value \\
\hline \multirow{5}{*}{ Control } & Similarities & 30 & $23 / 13$ & $3 / 11$ & $5 / 46$ & $0 / 05$ & $0 / 000$ \\
\hline & Information & 30 & $23 / 53$ & $3 / 48$ & $5 / 24$ & $0 / 05$ & $0 / 000$ \\
\hline & Block Design & 30 & $44 / 20$ & $3 / 01$ & $5 / 11$ & $0 / 05$ & $0 / 000$ \\
\hline & Picture Similarities & 30 & $16 / 40$ & $2 / 01$ & $2 / 43$ & $0 / 05$ & $0 / 018$ \\
\hline & IQ & 30 & $93 / 33$ & $9 / 37$ & $4 / 05$ & $0 / 05$ & $0 / 000$ \\
\hline \multirow{4}{*}{ Case } & Similarities & 30 & $18 / 80$ & $2 / 98$ & $5 / 46$ & $0 / 05$ & $0 / 000$ \\
\hline & Information & 30 & $18 / 73$ & $3 / 61$ & $5 / 24$ & $0 / 05$ & $0 / 000$ \\
\hline & Picture Similarities & 30 & $15 / 23$ & $1 / 69$ & $2 / 43$ & $0 / 05$ & $0 / 018$ \\
\hline & IQ & 30 & $83 / 60$ & $9 / 22$ & $4 / 05$ & $0 / 05$ & $0 / 000$ \\
\hline
\end{tabular}

All in all, it appears plausible that schizophrenic patients tend to experience higher level of cognitive problems in dealing with subtests of Wechsler Adult Intelligence test.

\section{Discussion}

Cognitive deficits are hallmark of schizophrenia and are considered as the central features of this disorder. In current study, we assessed malignant influence of this disorder on various subtests of Wechsler Adult Intelligence test. Accordingly, we found that schizophrenic tend to possess lower score comparing to healthy individuals. In a study conducted by Ritu and Colleagues (2015), it was concluded that cognitive markers like verbal memory deficits can distinguish unaffected siblings of schizophrenia from healthy controls and serve as an endophenotype for schizophrenia; the difference of our study is that we mainly focused on Wechsler subtests and various cognitive deficits and among adult patients [9]. In other studies conducted by Emre and Colleagues (2009), In 6 of 12 cognitive domains, people with schizophrenia performed worse than people with schizoaffective disorder or affective psychosis. However, the between-group differences were small and the distribution of effect sizes showed substantial heterogeneity. The betweengroup differences were driven by a higher percentage of males, more severe negative symptoms and younger age at onset of illness in the schizophrenia samples. In another study conducted by Guo and Colleagues (2011), it was concluded that impairment of cognitive function in schizophrenia patients with diabetes is severer than schizophrenia patients, suggesting that the prevention and management of diabetes may improve cognitive outcome in schizophrenia patients. In aforementioned study the authors focused on comorbid condition in schizophrenia and its role on severing cognitive deficits [12]. Patients with schizophrenia have been reported to perform worse than nonschizophrenic populations on neuropsychological tests, which may be affected by cultural factors. A study conducted by Haruo and Colleagues (2014), examined the performance of a sizable number of patients with schizophrenia on the Japanese version of the Wechsler Adult Intelligence Scale-III (WAIS-III) compared with healthy controls. The results indicate that the pattern and degree of impairment, as evaluated by the WAIS- III, in Japanese patients are similar to those previously reported in English-speaking patients and that the deficits of some neuropsychological domains relevant to functional outcomes are universally characteristic of schizophrenia [13]. Noble feature of current study is that in the present study, we independently replicated our previous cognitive classifications of people with schizophrenia. In addition, we extended previous work by demonstrating worse functional outcomes and symptom severity in the compromised group. The noble feature of this study is that we focused on specific cognitive deficits of schizophrenic patients; former studies mostly were focused on cognitive impairments in general sense. It appears plausible to focus on more specific cognitive impairments for future studies and try to differentiate and highlight cognitive impairments between schizophrenic patients and patients with comorbid conditions or other psychotic disorders.

\section{Authors' Contributions}

MBM and PS conceived and designed the evaluation. SMM collected and interpreted the clinical data and drafted the manuscript. All authors read and approved the final manuscript.

\section{References}

[1] John M. Grohol, Psy. D. (2014). DSM-5 Changes: Schizophrenia \& Psychotic Disorders. Available: http://pro.psychcentral.com/dsm-5-changes-schizophreniapsychotic-disorders/004336.html. Last accessed Aug 2016. 
[2] Ahbishekh Hulegar Ashok, John Baugh and Vikram K. Yeragani (2012). Paul Eugen Bleuler and the origin of the term schizophrenia (SCHIZOPRENIEGRUPPE). Available: http://www.ncbi.nlm.nih.gov/pmc/articles/PMC3339235/. Last accessed Aug2016.

[3] Erick Messias, MD, PhD, Chuan-Yu Chen, Ph. D and William W. Eaton, Ph. D. (2007). Epidemiology of Schizophrenia: Review of Findings and Myths. Available: http://www.ncbi.nlm.nih.gov/pmc/articles/PMC2727721/. Last accessed Aug2016. doi: 10.1016/j.psc.2007.04.007.

[4] Christopher R Bowie and Philip D Harvey. (Dec2006). Cognitive deficits and functional outcome in schizophrenia. Available:

http://www.ncbi.nlm.nih.gov/pmc/articles/PMC2671937/. Last accessed Aug2016.

[5] Mario FioravantiEmail author, Valentina Bianchi and Maria Elena Cinti. (2012). Cognitive deficits in schizophrenia: an updated metanalysis of the scientific evidence. Available: $\mathrm{https} / / / \mathrm{bmcpsychiatry.biomedcentral.com/articles/10.1186/147}$ 1-244X-12-64. Last accessed Aug2016.

[6] David Wechsler. (1939). Wechsler Adult Intelligence Scale Revised. Available: http://www.cps.nova.edu/ cpphelp/WAISR.html. Last accessed Aug2016.

[7] Perdu University. (2012). The Wechsler Intelligence Scales. Available: http://www.iupui.edu/ flip/wechsler.html. Last accessed Aug2016.

[8] Malakouti SK1, Fatollahi P, Mirabzadeh A, Zandi T.. (2007). Reliability, validity and factor structure of the GHQ-28 used among elderly Iranian.. Available:

http://www.ncbi.nlm.nih.gov/pmc/articles/PMC2671937/. Last accessed Aug2016.

[9] Ritu Nehra, Sandeep Grover, Sunil Sharma, Aditi Sharma, and Natasha Kate. (2016). Neurocognitive Functioning in
Schizophrenia, their Unaffected Siblings and Healthy Controls: A Comparison. Available: http://www.ncbi.nlm.nih.gov/pmc/articles/PMC4782445/. Last accessed Aug 2016. Indian J Psychol Med. 2016 Jan-Feb; 38 (1): 50-55. doi: 10.4103/0253-7176.175114.

[10] Emre Bora, Murat Yucel, Christos Pantelis. (2009). Cognitive functioning in schizophrenia, schizoaffective disorder and affective psychoses: meta-analytic study. Available: http://bjp.rcpsych.org/content/195/6/475. Last accessed Aug 2016. The British Journal of Psychiatry Nov 2009, 195 (6) 475-482; DOI: 10.1192/bjp.bp.108.055731.

[11] Ritu Nehra, Sandeep Grover, Sunil Sharma, Aditi Sharma, and Natasha Kate. (2016). Neurocognitive Functioning in Schizophrenia, their Unaffected Siblings and Healthy Controls: A Comparison. Available: http://www.ncbi.nlm.nih.gov/pmc/articles/PMC4782445/. Last accessed Aug 2016. doi: 10.3969/j.issn.16727347.2011.08.004.

[12] Guo X1, Zhang Z, Zhu W, Lian N, Lu H, Zhao J.. (2011). Cognitive functioning in schizophrenia with or without diabetes. Available:

http://www.ncbi.nlm.nih.gov/pmc/articles/PMC4782445/. Last accessed Aug2016. PMID: 21937796 DOI: 10.3969/j.issn.1672-7347.2011.08.004.

[13] Haruo Fujino, MS, Chika Sumiyoshi, PhD, Tomiki Sumiyoshi, MD, PhD, Yuka Yasuda, MD, PhD, Hidenaga Yamamori, MD, PhD, Kazutaka Ohi, MD, PhD, Michiko Fujimoto, MD, PhD, Satomi Umeda-Yano, Ph. (2014). Performance on the Wechsler Adult Intelligence Scale-III in Japanese patients with schizophrenia. Available: http://onlinelibrary.wiley.com/store/10.1111/pcn.12165/asset/p cn12165.pdf;jsessionid $=$ C0AF3A6F054A0A74C0FD85F4261 336A0.f01 $\mathrm{t} 02$ ? $\mathrm{v}=1 \& \mathrm{t}=$ isfwfj25\&s $=93182 \mathrm{cb} 52 \mathrm{f} 62 \mathrm{cb} 66 \mathrm{bbc} 864$ $525 \mathrm{~b} 69 \mathrm{e} 960 \mathrm{cc} 6 \mathrm{~b} 0 \mathrm{ce} 8 \&$ systemMessage $=$ Wil. Last accessed Aug2016, doi:10.1111/pcn.12165. 